\begin{tabular}{|l|l|l|l|l|}
\hline Revista Clío América & ISSN: 1909-941X & Vol. 9 & No. 18 & Julio - Diciembre de 2015 \\
\hline
\end{tabular}

\title{
Evolución de la cultura ciudadana: Análisis comparativo del caso de ciénaga, magdalena periodos 2011 y 2014
}

Evolution of civic culture: Comparative analysis ciénaga, magdalena's case periods 2011 and 2014

\section{Daniela Andrea Figueroa-González} Estudiante de Economía Universidad del Magdalena Santa Marta, Colombia mullerjuan15@gmail.com

Juan Mario Müller-Barrios Estudiante de Economía Universidad del Magdalena Santa Marta, Colombia danfiglez@gmail.com

Tipología:

Artículo de Investigación Científica y Tecnológica Fecha de Recibido: Junio 19 de 2015 Fecha de Aceptación: Agosto 03 de 2015

Para citar este artículo: Para citar este artículo: Figueroa, G. D., \& Müller, B. J. (2015). Evolución de la cultura ciudadana: análisis comparativo del caso de Ciénaga, Magdalena periodos 2011 y 2014. Clío América. 9 (18), 157 - 171
Resumen: Este artículo presenta los resultados obtenidos en el año 2011 y 2014, mostrando la evolución que ha tenido la cultura ciudadana del municipio de Ciénaga, Magdalena, a través de cuatro módulos que desarrollan temas como la convivencia, la cultura tributaria, la participación ciudadana, el uso de espacios públicos, entre otros; con miras a presentar los cambios en la conducta de los ciudadanos del municipio y con esto plantear una potencial política pública y educativa encaminada al fortalecimiento de la cultura ciudadana.

Palabras clave: Cultura, democracia, participación, ciudadanía

JEL: A1, D74, R11, Z13

Abstract: This article presents the results obtained in 2011 and 2014, showing the evolution that has taken the civic culture of the municipality of Ciénaga, Magdalena, through four modules that develop topics such as coexistence, the tax culture, citizen participation, use of public spaces, among others; with a view to making changes in the behavior of citizens in the municipality and thereby pose a publiceducation aimed at strengthening citizen potential political culture.

Keywords: Culture, democracy, participation, citizenship 


\section{Introducción}

Las normas, costumbres y formas de vida evolucionan constantemente con los seres que componen una sociedad o comunidad, la importancia de tener claros los lineamientos en los que se basa la convivencia, los comportamientos que se consideran como indeseables, y hasta la percepción que tiene la población sobre sus dirigentes, son importantes a la hora de definir aquello que permita proteger los intereses comunes y procurar el bienestar común a través de la convivencia pacífica.

Cada espacio habitado tiene diferencias tanto geográficas, como en la composición y características comunes de sus habitantes, lo que forma las bases de la cultura, que no es más que las conductas, 0 lo que se considera en común como bueno o malo, que se da a conocer al resto de las culturas a través de las formas de expresión cultural, las costumbres y los rituales que se practican (CORPOVISIONARIOS).

El municipio de Ciénaga no es la excepción a esta conducta humana, por lo tanto, se ha realizado un estudio de corte transversal que compare la conducta de la ciudadanía y sus percepciones en temas como cultura tributaria, uso de los espacios públicos, convivencia, movilidad, para año 2011 y el 2014.

El estudio cobra relevancia dado que a partir de estos lineamientos se pueden identificar e implementar acciones que permitan fortalecer las relaciones con el gobierno local y las dinámicas entre los mismos habitantes del municipio. Cabe resaltar que debido a la metodología utilizada para la obtención de los resultados no es posible explicar el comportamiento de las variables, ni mucho menos establecer relaciones entre ellas, sin embargo, cada componente evaluado será descrito presentando una comparación de los datos obtenidos en el año 2011 y en 2014, que sirvan de base para futuras investigaciones.

El artículo se desarrolla en seis secciones, en la primera se hace una breve introducción sobre el tema y se explica el objetivo de este estudio; en la segunda se hace una revisión literaria que incluye diversos estudios de caso aplicados en otras ciudades; en la tercera se explica la metodología bajo la que se desarrolló la investigación; en la cuarta sección, se presentan los resultados obtenidos; en la quinta se concluye; y por último, en la discusión se indican los inconvenientes en el desarrollo del texto, los aportes al conocimiento del área y los lineamientos a seguir en las futuras investigaciones.

\section{Estado del arte}

En materia de cultura ciudadana, se realizó una revisión a nivel nacional e internacional, entre estos se encuentra el trabajo hecho por Mockus (2001) quien planteó la hipótesis del divorcio entre ley, moral y cultura en miras de sistematizar la experiencia de la alcaldía de Bogotá (1995-1997) referente a acciones y resultados de prevención y control de violencia. El trabajo buscó medir la fuerza de autonomía y autorregulación cultural al dividir estos tres sistemas reguladores del comportamiento, y así determinar la incidencia del contexto social y cultural sobre estos comportamientos. La finalidad era resolver la problemática de la seguridad viéndolo desde el punto de vista de la convivencia, trayendo consigo un nuevo enfoque y presentando además coherencia entre la regulación moral, cultural y jurídica.

Por su parte Illera (2005) integró la relación entre convivencia ciudadana y derecho de policía bajo un marco teórico-conceptual en la ciudad de Barranquilla, en el que la cultura ciudadana fue considerada como la base del buen convivir entre individuos, en este sentido, es la policía quien debe velar por que haya total cumplimiento de las reglas jurídicas que garantizan la existencia de los valores colectivos basados en principios totalmente constitucionales. Concluyó que existen reglas bajo un sistema de creencias y costumbres que son limitantes y que permiten que haya orden social a través de la regulación cultural.

En el marco del programa de Visión Colombia 2019, se consideró que la acción que promueve el Estado en materia de brindar paz duradera y seguridad a los ciudadanos es algo limitado, pues esto solo se garantiza con la ayuda de la ciudadanía en general, esta colaboración que permite que puedan responsabilizarse no solo de la seguridad individual, sino de la colectiva, estimando que la colaboración de las autoridades esté presente también. Asimismo, es importante que todos los individuos compartan en 
los diferentes escenarios sus actitudes y que estas vayan acorde a un comportamiento respetuoso bajo las leyes y normas de convivencia ya establecidas, que generen ambientes de total respeto y que a su vez sea promovida la confianza entre la comunidad y las autoridades para que finalmente esto responda a principios de una buena democracia (Departamento Nacional de Planeación -DNP-, 2006).

Por su parte Rincón (2006) piensa que el tema de cultura ciudadana está arraigado a trabajos sociales, que permitan conocer la existencia de nuevos elementos que contribuyen a la redefinición de la nueva ciudadanía, el estudio abordó las principales nociones de ciudadanía a lo largo de los años, enmarcando el tema de cultura ciudadana desde tres propuestas, principalmente postuladas por Mockus en 2001, quien hace referencia a que sociólogos entre los 60 y los 70 buscaron actualizar teorías relacionadas con la reproducción cultural basados en principios de reproducción productiva y relaciones económicas, con resultados desfavorables al no obtener los cambios esperados en cuanto a reproducción cultural, al no estar subordinado por los fenómenos económicos (Rincón S., 2006).

Guillen, Sáenz, Badii, y Castillo (2009) detallaron el por qué los individuos participan en procesos democráticos relacionados con la ciudadanía en los que las personas se involucran activamente, dada la importancia que representa este aspecto en la vida del ser humano y que viene relacionada con el desarrollo de las personas, destacando valores incluidos en este acto como la solidaridad, humanismo, tolerancia, etc., y que le infunden al ser humano un despertar en la búsqueda del fin específico que mejore los niveles de vida en una forma individual y a su vez colectiva. Así mismo, los autores rescatan la idea que la participación ciudadana es un derecho de todas las sociedades y que sin embargo hay quienes no saben guardarlo bien, por tanto ellos creen que por vivir en la modernidad es un deber hacer funcionar esas libertades que propician un espacio donde la democracia es el eje principal del mundo.

En un estudio, Ruiz y Trucios (2009) afirman: "demostrar los niveles de miedo al delito, victimización y cultura ciudadana en una muestra de estudiantes universitarios de cinco países, Argentina, Colombia, El Salvador, España y Perú" (p. 193). Encontraron a través de matrices de correlación que la cultura ciudadana está asociada primordialmente con el nivel de satisfacción que sienten los individuos con el servicio que ofrece la policía en el tema de seguridad, al menor temor y al delito ente otros.

Rojo (2009) realizó un análisis de casos de estudio, con el fin de evidenciar que a través de una buena cultura ciudadana se puede optimizar y garantizar el buen uso y funcionamiento adecuado de los servicios públicos, destacando que el estado es el garante de brindar estos, pero que no es el único responsable de su buen uso y mantenimientos. Concluyó que una buena cultura ciudadana en la actividad y manejo de los servicios públicos garantiza el funcionamiento duradero y óptimo a futuro, puesto que si las personas crean espacios de buena convivencia y manejo de las normas del ciudadano, lo más probable es que el desarrollo de una ciudad se logre en todos los ámbitos.

Por su parte, Zambrano (2010) desarrolló aspectos conceptuales desde una perspectiva amplia para poder analizar la relación entre los tres componentes que utiliza en su estudio. Además de esto, el autor, establece que deben existir retos que permitan generar un nuevo ambiente de gestión cultural deducida de la constitucionalización de ciertos derechos relacionados con la cultura, debido a que son quienes generan un ambiente renovador en el sector cultural.

En 2010, un trabajo realizado por Ruiz estudió las posibles relaciones entre la eficacia colectiva, la cultura ciudadana y varias mediciones del miedo al crimen (Ruíz, 2010, p. 103), obteniendo como resultados una alta correlación entre sí, demostró que una pequeña parte de esa información muestra claramente la unión existente entre eficacia colectiva y menores niveles de delito, esto es debido al ámbito socio-geográfico, estableciendo que el miedo al delito viene arraigado a ese riesgo al cual puede incurrir un individuo y los temores que vienen acompañados en los espacios geográficos en los que se podrían encontrar es decir barrios, localidades y ciudad. 


\section{Metodología}

Basados en la metodología propuesta por Hernández, Fernández y Baptista (2004) sobre la clasificación de estudios de ciencias sociales, este trabajo de investigación es considerado de tipo descriptivo, pues como su nombre lo indica, se describen situaciones, eventos y hechos, que permitan especificar las propiedades, las características, los perfiles importantes de personas, grupos, comunidades o cualquier otro fenómeno que se someta a un análisis (Danke, 1989, citado por Hernández, Fernández \& Baptista, 2004), en este caso, la cultura ciudadana en el municipio de Ciénaga. Así mismo, esta metodología de estudio se desarrolla por medio de la realización de preguntas y entrevistas a las personas que pertenezcan a los grupos focales de interés para la investigación proporcionando información primaria.

\section{a. Sujeto de Estudio}

Se realizó una encuesta a la población en edades a partir de los 14 años que se encontraban en lugares específicos como iglesias (católicas y evangélicas), colegios, hospitales, plazas y parques, mercado y centro, que representan los lugares con mayor interacción entre los habitantes del municipio, lo que permitió tener diferentes puntos de vista sobre un mismo tema.

\section{b. Tamaño de la Muestra}

Para el censo realizado en el año 2005, el municipio de Ciénaga tenía una población total de 101.985 habitantes en la zona urbana (DANE 2014), de estos el $49,5 \%$ son hombres y $59,5 \%$ mujeres (ver tabla 1 ).

Por lo tanto, al momento de la realización de la encuesta, se tuvo en cuenta la distribución demográfica por género, encuestando mayor cantidad de mujeres y hombres en edades entre los 20 a los 40 años.
Tabla 1.

\section{Porcentaje de la población urbana de Ciénaga Magdalena por Sexo}

\begin{tabular}{|c|c|c|}
\hline $\begin{array}{c}\text { POBLACIÓN / } \\
\text { GÉNERO }\end{array}$ & HOMBRE & MUJERES \\
\hline Urbana & $49,5 \%$ & $59,5 \%$ \\
\hline
\end{tabular}

Fuente: Censo DANE 2005.

La fórmula utilizada para determinar el tamaño de la muestra en el municipio de Ciénaga fue la siguiente:

Ecuación $1 n_{0}=\frac{z^{2} * p q}{e^{2}}$

Dónde:

$z=$ Es un factor probabilístico que viene dado por el nivel de confianza que se decida trabajar, en este caso, para un nivel de confianza de $95 \%$, el valor de $z$ asignado es 1,96 .

$p q=$ Varianza de la proporción; $p=0,05 ; q=0,95$.

$\mathrm{e}=$ Error permitido; 0,06732

\section{Ajustándolo al número de la población conocida:}

$$
\text { Ecuación } 2 \boldsymbol{n}^{*}=\frac{\boldsymbol{n}_{0}}{1+\frac{\left(n_{0}-1\right)}{N}}
$$

Dónde:

$\mathrm{N}=$ Población total; $\mathrm{N}=104.060^{1}$

Ecuación $1.1 n_{0}=\frac{(1,96)^{2} *(0,05 * 0,95)}{(0,06732)^{2}}=211,91$

Ecuación $2.1 n^{*}=\frac{211,91}{1+\frac{(211,91-1)}{104.060}}=211,48 \approx 211$ 


\section{c. Instrumento}

Par este estudio en particular se decidió utilizar como instrumento de recolección de los datos, una encuesta tipo cuestionario, basados en la encuesta realizada en 2011 en el Estudio de Cultura Ciudadana en Ciénaga Magdalena (Rico, 2011), con el fin de obtener los datos necesarios para realizar las respectivas comparaciones.

El instrumento fue modificado teniendo en cuenta las condiciones actuales. La encuesta consta de cuatro (4) módulos:

a) Uso de la ciudad, convivencia, legalidad y participación ciudadana

b) Justicia y Cultura Democrática

c) Cultura tributaria

d) Deporte, recreación y cultura

\section{Resultados}

Los resultados del estudio de investigación en el municipio de Ciénaga, Magdalena, permitió constatar cuáles cambios hubo en cultura ciudadana en los habitantes del municipio desde el 2011y 2014,

En cuanto al módulo uso de la ciudad, convivencia, legalidad y participación ciudadana, se estableció que con respecto a la convivencia los habitantes del municipio siguen reacios en cuanto a convivir con personas drogadictas; sin embargo, es posible afirmar que en cuanto a los resultados obtenidos en el año 2011, su tolerancia frente a los homosexuales, reinsertados y políticos ha disminuido.

\section{Figura 1.}

\section{Personas nos deseadas en los vecindarios}

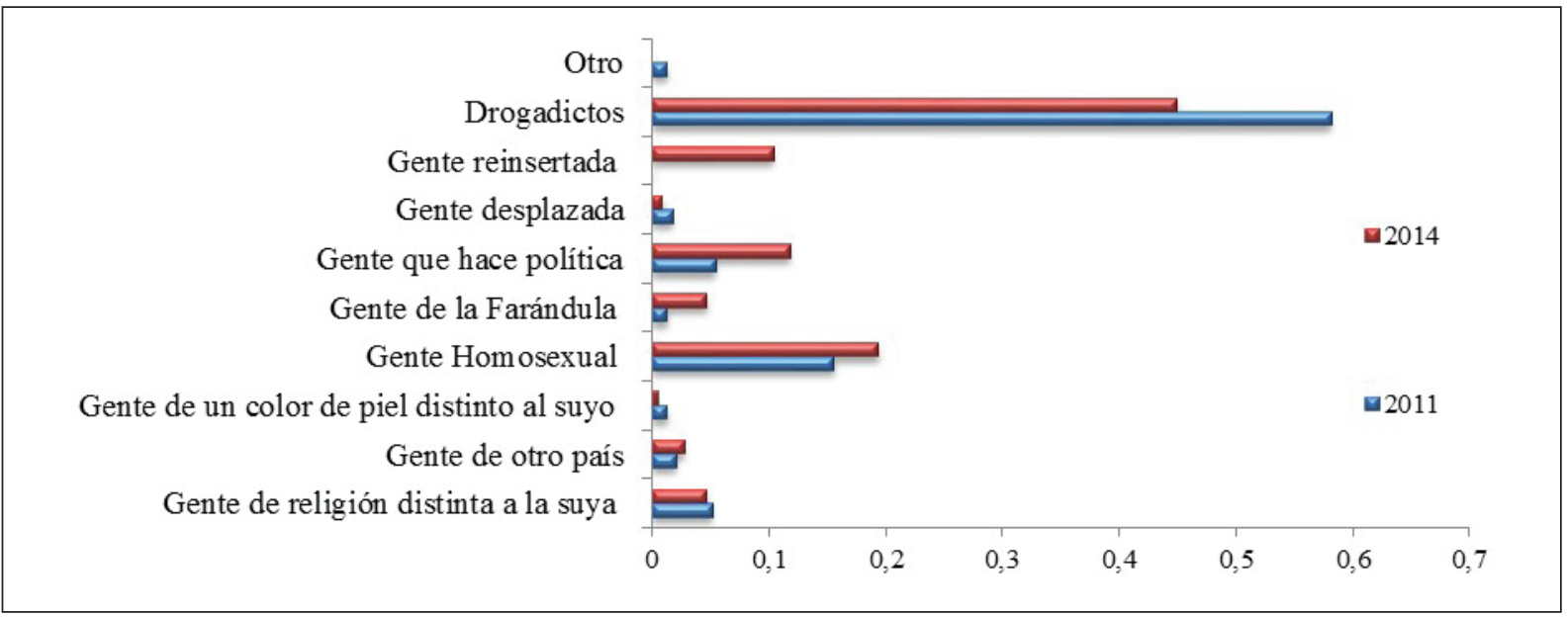

Fuente: Encuestas de cultura ciudadana en Ciénaga, Magdalena 2011 y 2014. 
En cuanto a movilidad, se percibió cambios de preferencia del ciudadano de Ciénaga, dado que este dejó de transitar por las calles a pie como lo hacía para el año 2011, para movilizarse en motocicleta en el 2014, evidenciando que este vehículo ha sido la principal opción por parte de las personas debido a que ahorra tiempo y permite un ágil desplazamiento sobre las vías del municipio.

\section{- Figura 2.}

\section{Principal medio de transporte}

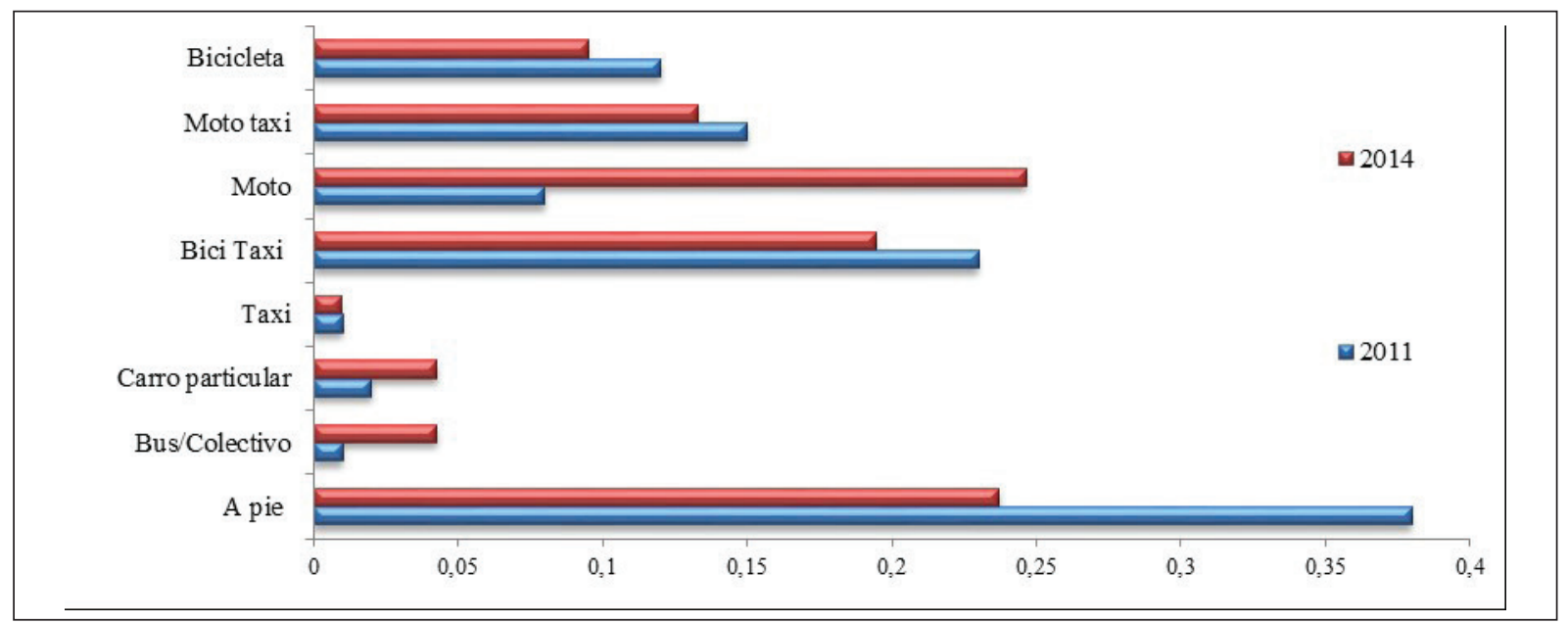

Fuente: Encuestas de cultura ciudadana en Ciénaga, Magdalena 2011 y 2014.

En temas de espacio público, ambos estudios revelaron que el cienaguero promedio, sin importar su nivel educativo, reconoce de manera precisa el concepto de espacio público, como se observa en la figura 3.

\section{Figura 3.}

\section{Percepción de los espacios públicos}

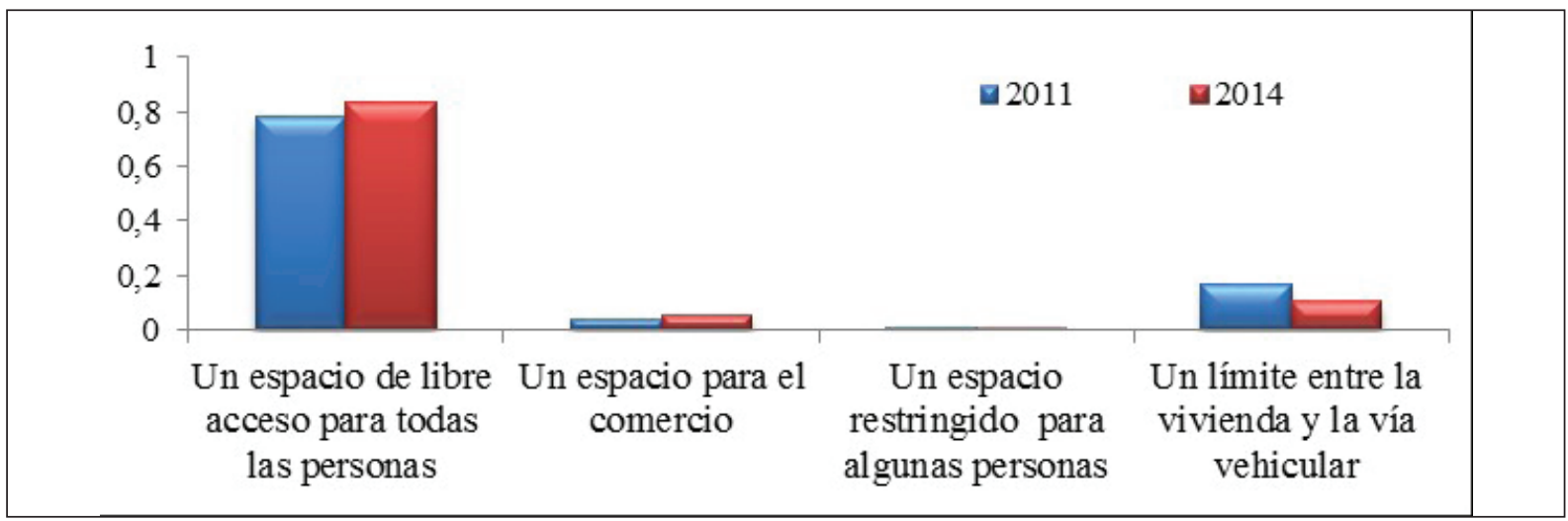

Fuente: Encuestas de cultura ciudadana en Ciénaga, Magdalena 2011 y 2014. 
El espacio público más concurrido por la comunidad, son los andenes peatonales; sin embargo, frente a los resultados obtenidos en 2011, el ciudadano promedio prefiere en el 2014 la utilización de plazas y parques.

\section{Figura 4.}

\section{Espacios públicos más utilizados}
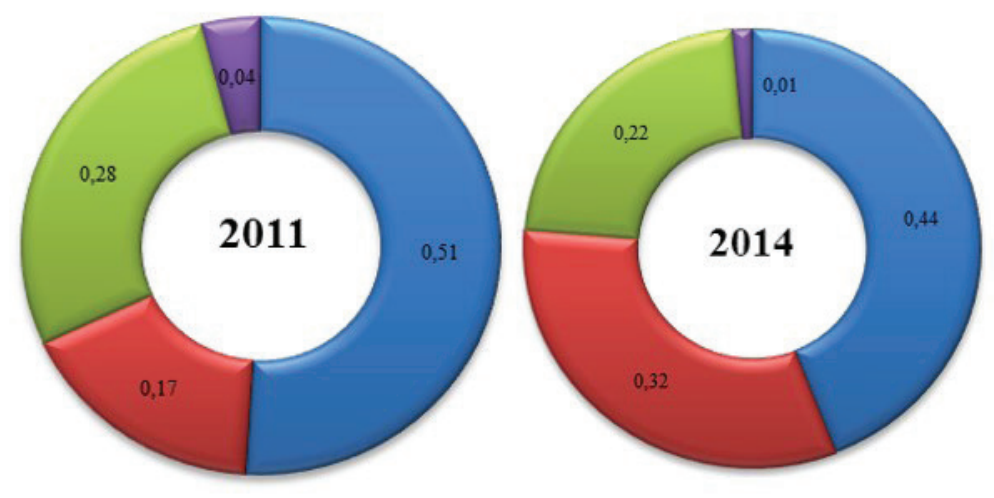

Andenes peatonales

Plazas y parques

Vía vehicular

Otro

Fuente: Encuestas de cultura ciudadana en Ciénaga, Magdalena 2011 y 2014.

Adicionalmente se encontró que lo que más le genera incomodidad a los habitantes es la violación de decibeles sonoros permitidos en áreas residenciales a causa de los equipos de sonido a alto volumen más conocidos como pick up, lo que trae consigo malestar acústico, este resultado difiere al del año 2011 en el que los encuestados determinaron que el arrojar basuras era lo que más indignaba a la ciudadanía.

\section{Figura 5.}

\section{Comportamientos dañinos para el municipio de Ciénaga, Magdalena}

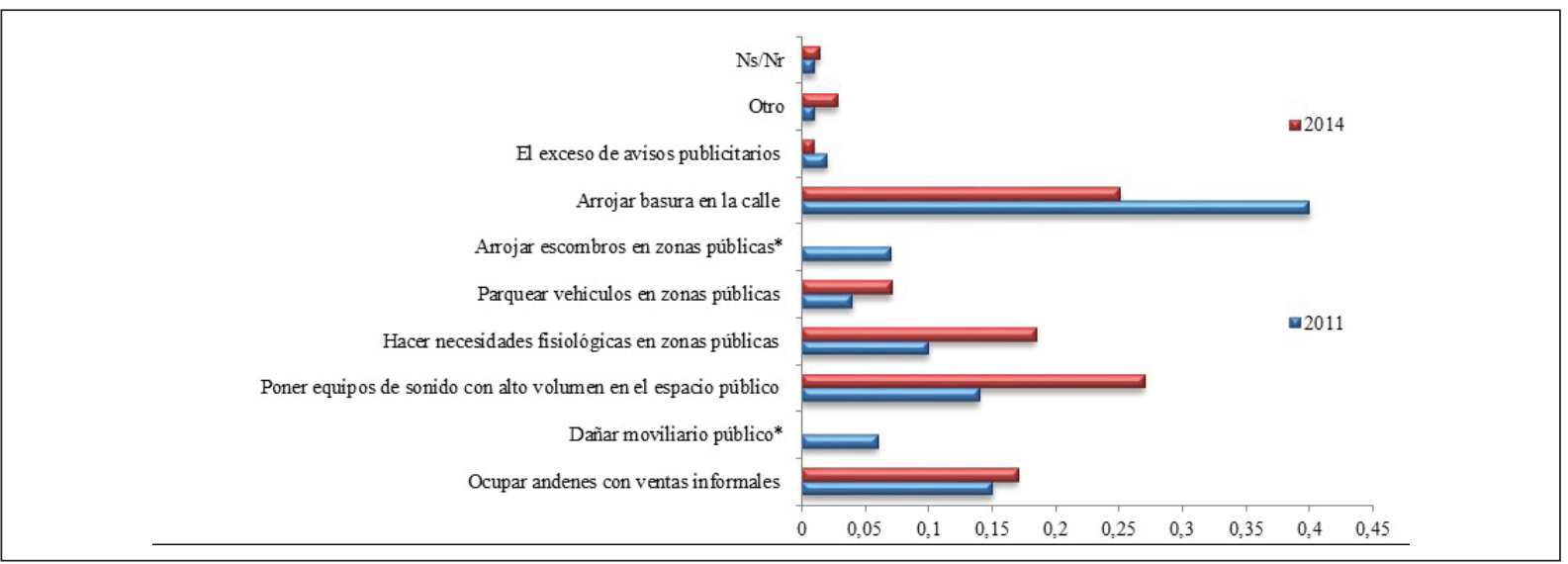

Fuente: Encuestas de cultura ciudadana en Ciénaga, Magdalena 2011 y 2014. 


\section{- Figura 6.}

\section{Porcentajes de víctimas de falsificación 0 fraude}

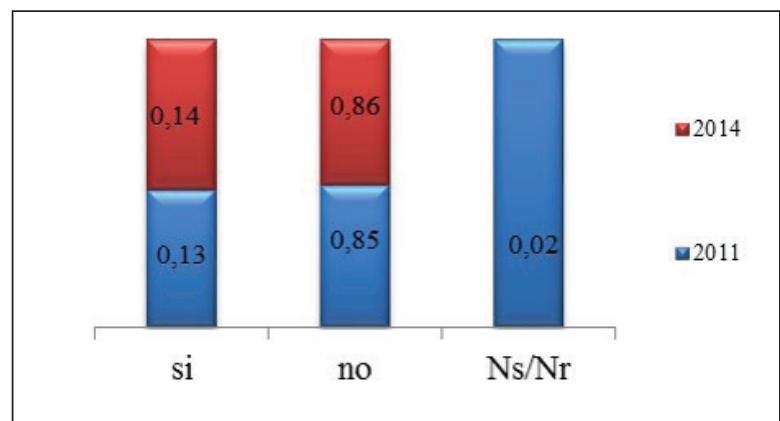

Fuente: Encuestas de cultura ciudadana en Ciénaga, Magdalena 2011 y 2014.

\section{Figura 7.}

\section{Conocimiento de fraudulencia en la conexión de servicios públicos en el vecindario}

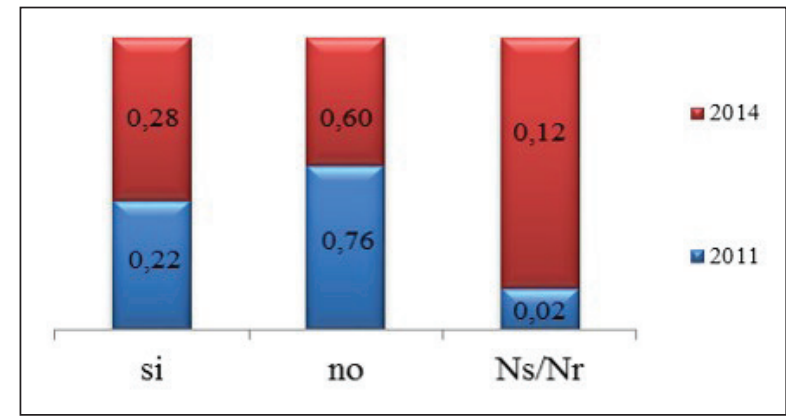

Fuente: Encuestas de cultura ciudadana en Ciénaga, Magdalena 2011 y 2014.

Por otra parte, se evidenció a través del estudio realizado que el municipio con relación a la legalidad, presenta un porcentaje reducido en cuanto a víctimas de fraude; sin embargo aún se presentan casos, lo que demuestra que los habitantes del municipio no son ajenos a este tipo de hechos.

Asimismo se identificó que los encuestados en su mayoría admitieron no conocer casos de manipulación de las redes de servicios públicos por otros individuos, los gráficos 6 y 7 evidencian lo dicho anteriormente.

En relación a la justicia y la cultura democrática se pudo identificar que los ciénagueros conservan un pensamiento escéptico cuando se les habla de política, paradigma que se ha creado en torno a los escándalos en los que se han visto involucrados las personas que participan en ella, lo que ha generado en la ciudadanía un descontento que ha contribuido a que los 'políticos' no tengan credibilidad ante la sociedad.

Figura 8.

\section{Percepción de la palabra " Política"}

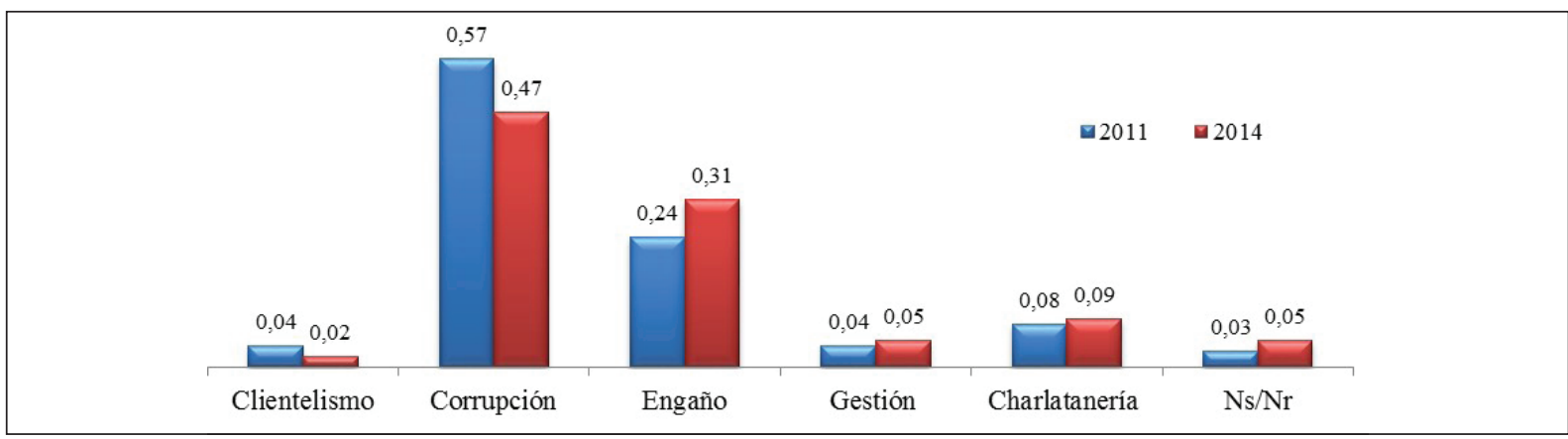

Fuente: Encuestas de cultura ciudadana en Ciénaga, Magdalena 2011 y 2014. 
Evidenciando con esto que la palabra 'política' la familiarizan mucho con los términos corrupción y engaño, se podría decir que en el municipio existe una apatía hacia estos, y que probablemente los ciénagueros tienen esta clase de pensamientos por el actuar de los pasados gobernantes del municipio.

Figura 9.

\section{Participación electoral}



Fuente: Encuestas de cultura ciudadana en Ciénaga, Magdalena 2011 y 2014.

Sin embargo en los resultados obtenidos en la investigación del 2014 y 2011, los cienagueros siguen creyendo que la participación de los ciudadanos en la toma de decisiones políticas contribuiría a que existiese más democracia. Además, se denota que cuando es tiempo de elecciones los habitantes de Ciénaga participan activamente, ejerciendo su derecho al voto como buenos ciudadanos.

Figura 10.

Percepción de la participación de la ciudadanía en la toma de decisiones políticas

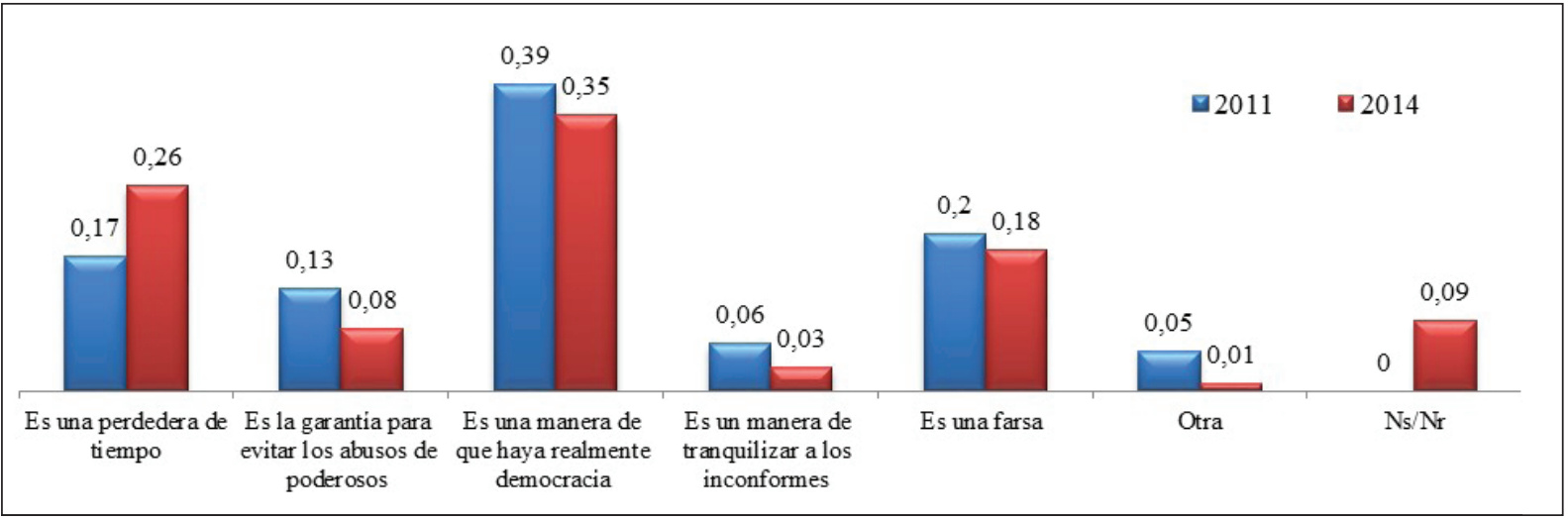

Fuente: Encuestas de cultura ciudadana en Ciénaga, Magdalena 2011 y 2014. 
Por otro lado, en materia de cultura tributaria el ciudadano tiene claro por qué se debe tributar, y además la comunidad de Ciénaga cumple efectivamente con sus deberes al cancelar los impuestos ante el municipio; es interesante ver que la población juvenil del total de los encuestados y en ambos estudios son quienes desconocen por completo por qué razón se debería cancelar impuestos.

\section{- Figura 11.}

\section{Conocimiento de los conceptos por los cual se paga impuestos}

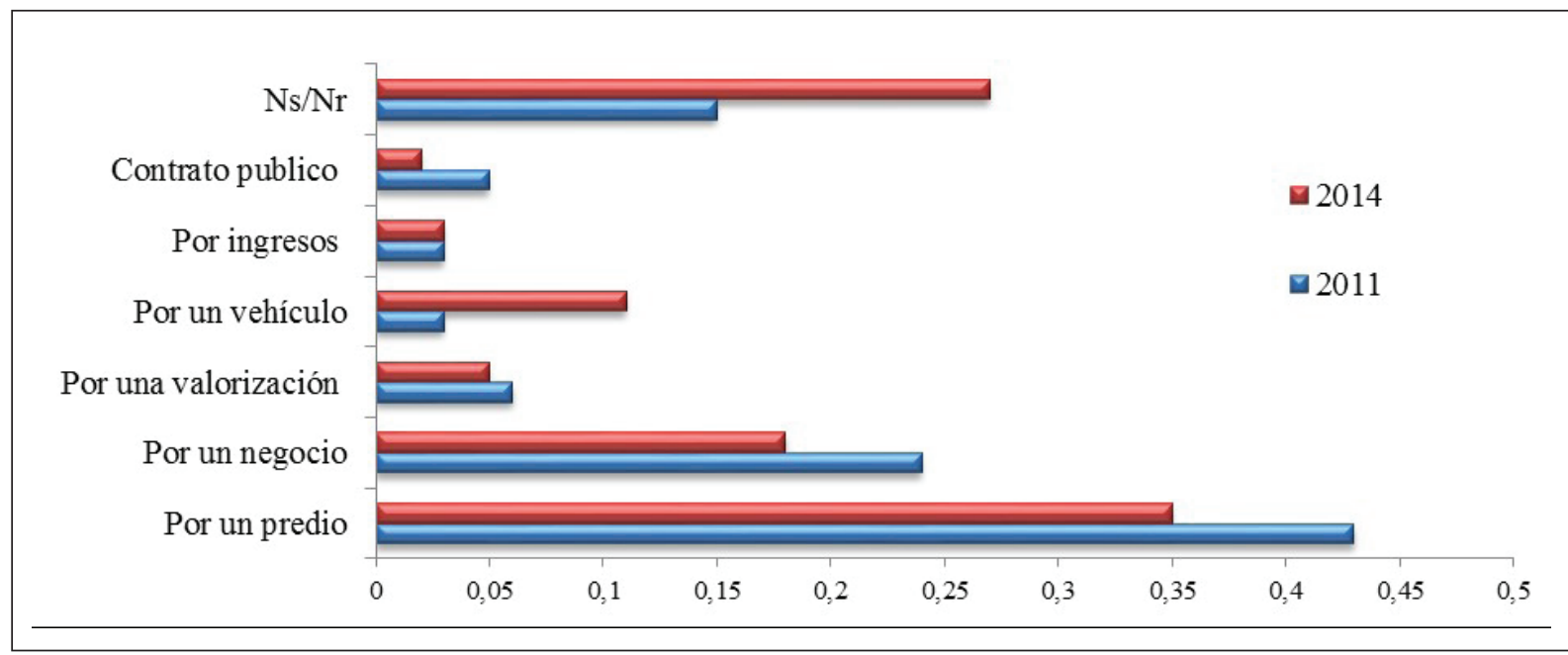

Fuente: Encuestas de cultura ciudadana en Ciénaga, Magdalena 2011 y 2014.

En este sentido, se indagó a los ciudadanos en qué le gustaría que se invirtieran los impuestos del municipio y evidentemente se reafirma que desearían que fuese en el sector salud. Los resultados obtenidos en el 2014 constatan que el sistema de salud en el municipio es deficiente y no subsana toda la demanda que hay actualmente. Además hay quienes piensan que no hay suficientes controles en Ciénaga para evitar que sus recursos sean desviados a manos inescrupulosas, lo que deja como resultado que la desconfianza existente hacia quienes realizan política sea bastante significativa.

Por último, en deporte, recreación y cultura es curioso ver que si bien el municipio goza de una oferta cultural variada tanto en los estudios del 2011 y 2014, los resultados de los encuestados frente a este tema demuestran que siguen desconociendo cuál es el significado de la palabra 'cultura', y que para ambos años de estudio se sigue demostrando que los ciudadanos ignoran cuál es la oferta cultural que les ofrece su municipio. Esto basado en los porcentajes obtenidos producto de ambas investigaciones en lo que se ve que no ha habido cambio alguno con respecto a este tema, algo paradójico si se compara cuando se indagó sobre su participación en la oferta cultural, a lo que respondieron en mayor proporción del total de encuestados, que sí admiten asistir a esta clase de festejos, sin embargo el nivel de participación de la oferta cultural que ofrece el municipio disminuyó con respecto al 2011. 


\section{-jFigura 12.}

\section{Preferencia del área o sector de inversión de los impuestos}

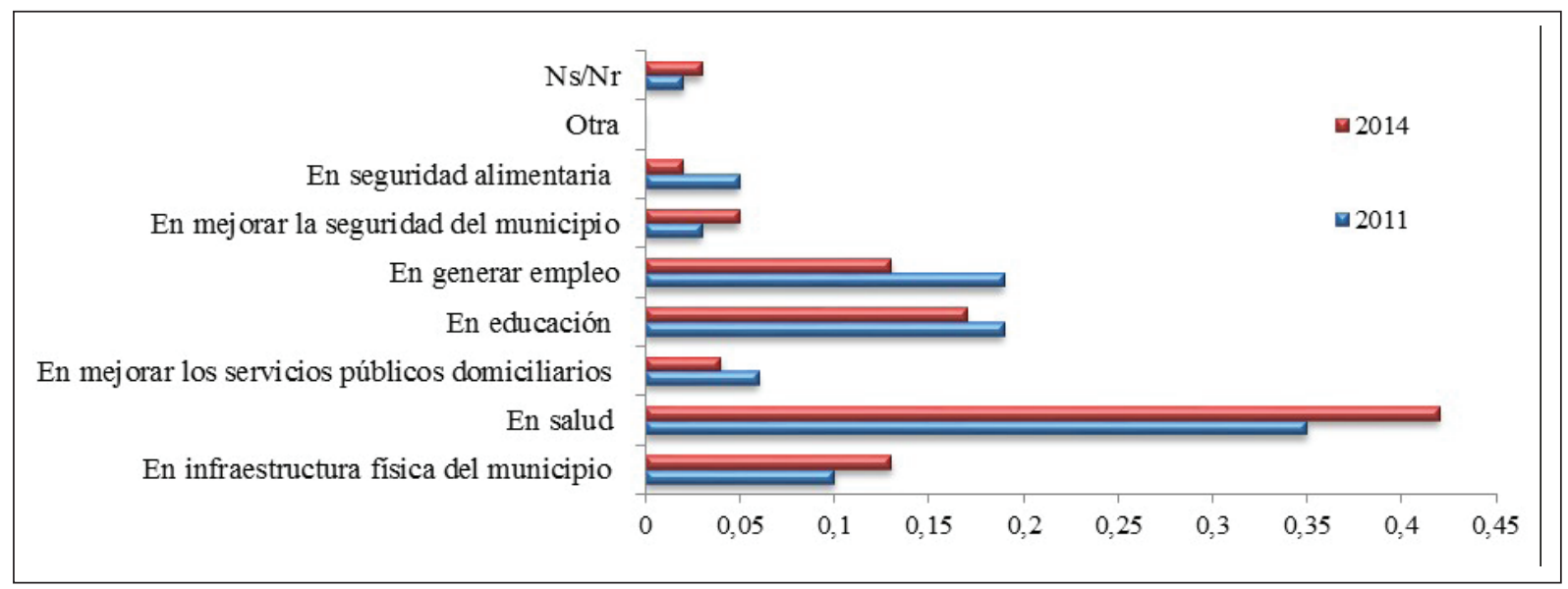

Fuente: Encuestas de cultura ciudadana en Ciénaga, Magdalena 2011 y 2014.

Figura 13.

\section{Conocimiento de la oferta cultural de Ciénaga}

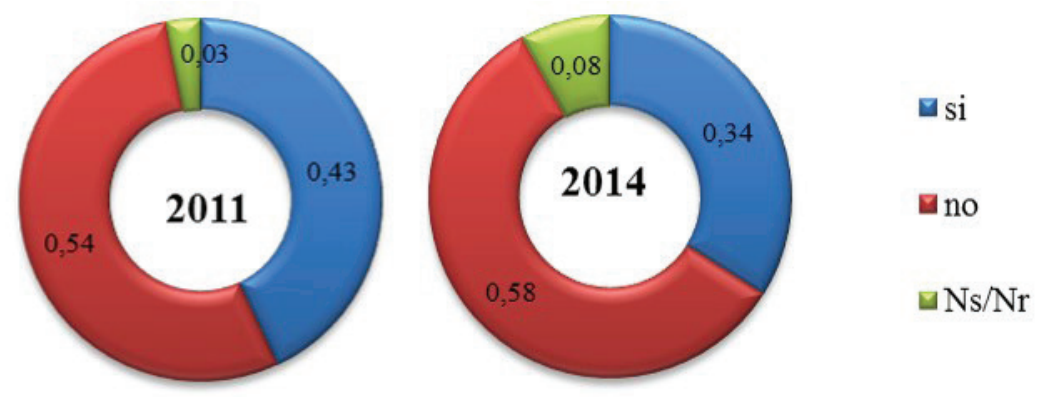

Fuente: Encuestas de cultura ciudadana en Ciénaga, Magdalena 2011 y 2014.

Dando a conocer que la fiesta que los caracteriza a ellos como cienagueros ha sido ignorada por los propios, estas festividades que deberían abarcar todas las tradiciones de antaño se ha vuelto en una algarabía en la que solo es prestada para ser centro de bailes, dejando a un lado el valor intangible que les ha dejado ese legado que llevan como habitantes de Ciénaga. 


\section{Figura 14.}

\section{Participación de la oferta cultural del municipio}
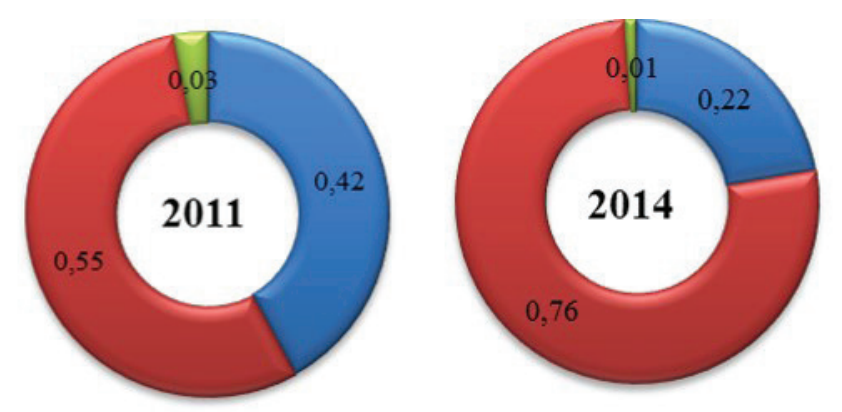

$\square \mathrm{si}$

घno

$\square \mathrm{Ns} / \mathrm{Nr}$

Fuente: Encuestas de cultura ciudadana en Ciénaga, Magdalena 2011 y 2014.

\section{Figura 15.}

\section{Participación de la ciudadanía en la oferta cultural de Ciénaga, Magdalena}

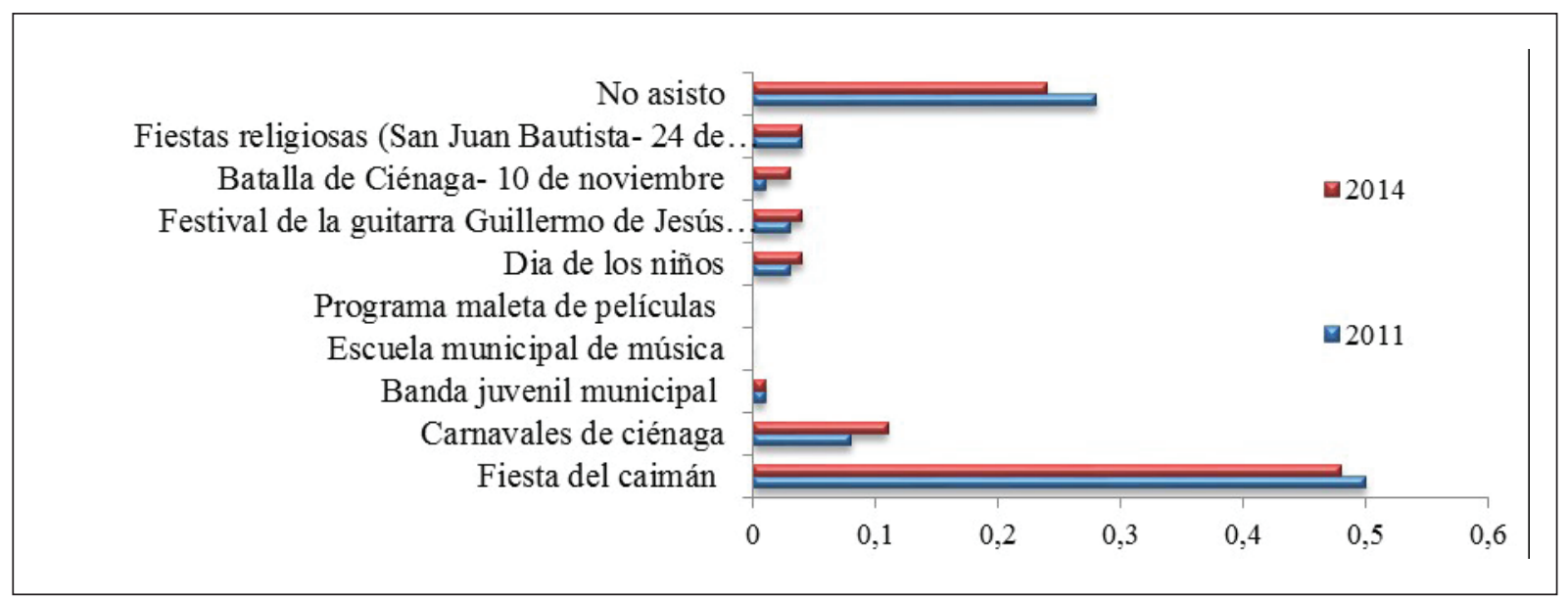

Fuente: Encuestas de cultura ciudadana en Ciénaga, Magdalena 2011 y 2014. 


\section{- Figura 16.}

\section{Importancia de los patrimonios culturales de Ciénaga, Magdalena}

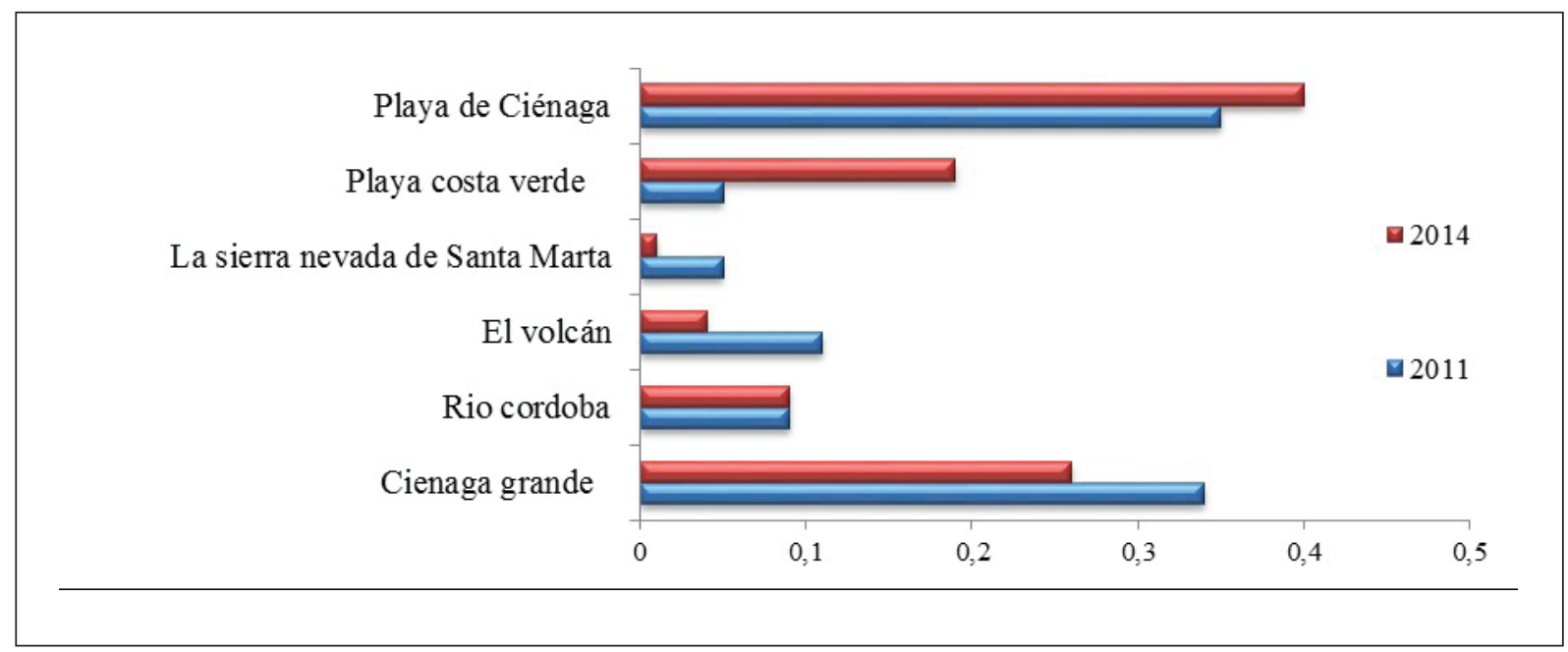

Fuente: Encuestas de cultura ciudadana en Ciénaga, Magdalena 2011 y 2014.

Además, los ciudadanos encuestados en 2014 consideraron que para ellos tiene mayor relevancia como patrimonio cultural la playa del municipio, como se puede observar en la figura 16, dejando a un lado lugares exóticos como la Ciénaga Grande, que además de su magnificencia es generadora de empleo para las pequeñas colonias de pescadores que subsisten en esta área.

Adicionalmente se determinó que el habitante común de Ciénaga no es dado al deporte, probablemente no exista una buena cultura deportiva dentro del municipio, por el estado de deterioro o la inexistencia de espacios en los que las personas pueden trabajar físicamente cualquier clase de actividad deportiva, esto se logra observar en ambos estudios donde el ciudadano argumenta que es muy deficiente la infraestructura para poder ejercer un excelente desarrollo del deporte y los que hay no cumplen con las condiciones adecuadas para trabajar en ellos.
HFigura 17.

\section{Porcentaje de personas que practican algún deporte}

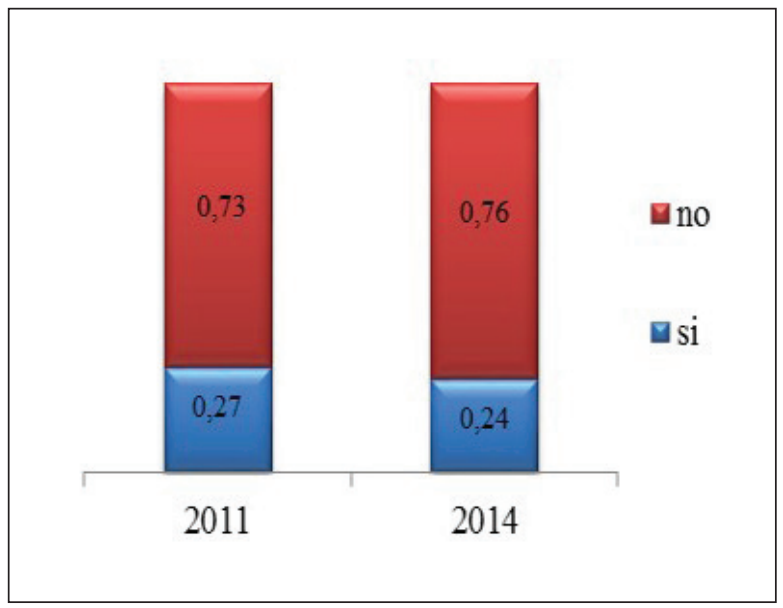

Fuente: Encuestas de cultura ciudadana en Ciénaga, Magdalena 2011 y 2014. 
Figura 18.

Percepción de los escenarios deportivos en Ciénaga, Magdalena

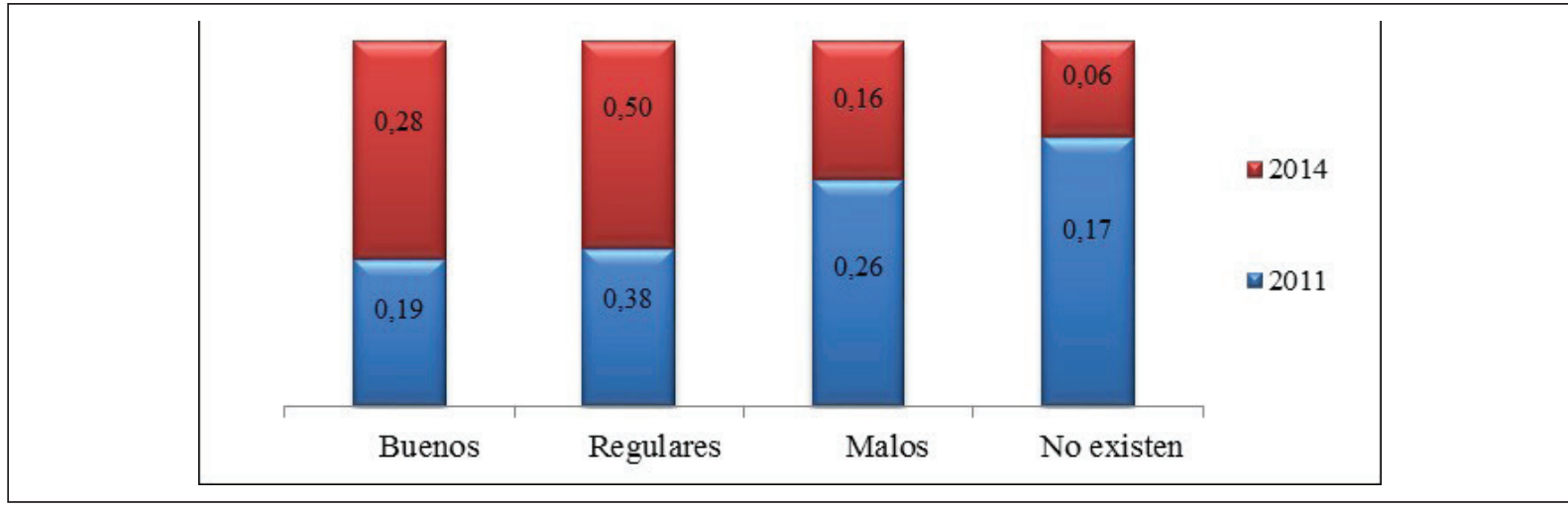

Fuente: Encuestas de cultura ciudadana en Ciénaga, Magdalena 2011 y 2014.

\section{Conclusión}

Una vez hecho el análisis comparativo se ha constatado que la ciudadanía aún presenta deficiencias en materia de convivencia ciudadana, legalidad, cultura tributaria, oferta cultural y deportiva y cultura democrática, debido a que no se tiene claridad de las normas de convivencia, los deberes y derechos del ciudadano, que son herramientas básicas que deben ser implementadas desde el hogar y fomentadas en la escuela.

Es necesario crear políticas y estrategias educativas encaminadas al fortalecimiento de valores como: respeto, tolerancia, honestidad entre otros; los cuales contribuyen a construir buenas prácticas para establecer en las actuales generaciones agentes formadores de una cultura ciudadana.

Como conclusión general, no se evidencia una evolución positiva de la cultura ciudadana en el municipio, es por esto que se debe primero hacer una campaña educativa de sensibilización, que enseñe a los ciudadanos más jóvenes a cumplir con las normas de convivencia elementales, que aprendan por qué se debe tributar, qué es un tributo y a qué áreas del municipio se deben destinar estos dineros, cuál es la oferta cultural que el municipio ofrece, y los patrimonios naturales que son únicos de la región. Así mismo, una política pública robusta destinada a la lucha contra la corrupción y la desviación de dineros del Estado, y a la recuperación de áreas comunes y la percepción de seguridad de la ciudadanía.

\section{Discusión}

Aunque Ciénaga es el municipio con mayor extensión poblacional del departamento del Magdalena y recibe en regalías por concepto de 20 proyectos aprobados por valor de $\$ 78.373 .490 .247$ de pesos (DNP, 2015), aún se pueden ver rezagos culturales frente a otros municipios que cumplen las mismas características como lo son Soledad en Atlántico, Aguachica en Cesar y Soacha en Cundinamarca.

En el desarrollo de la investigación fue posible encontrar vacíos en la literatura acerca del tema, lo que impidió enriquecer el conocimiento y las experiencias de otras ciudades en esta área del conocimiento antes de la puesta en marcha del trabajo de campo. Sin embargo fue posible constatar durante el proceso de observación de la evolución de la cultura ciudadana en el municipio de Ciénaga, Magdalena que no ha habido cambios significativos en el comportamiento de los ciudadanos en lo que refiere a cultura y ciudadanía.

A pesar de las limitaciones encontradas, es necesario continuar el estudio de la cultura ciudadana en las distintas ciudades y municipios del país, sin embargo se recomienda a futuro la integración disciplinar entre psicólogos, sociólogos, antropólogos 
y demás estudiosos de las ciencias sociales para lograr un resultado más completo sobre el actuar de los individuos.

En este sentido, el estudio debe ser realizado anualmente y a familias determinadas para ver los posibles cambios frente a temas específicos o luego de la aplicación de campañas y planes encaminados al fortalecimiento de la cultura.

\section{Referencias Bibliográficas}

Departamento Nacional de Planeación -DNP-. (2006). Fomentar la cultura ciudadana.

Guillen, A., Sáenz, K., Badii, M., \& Castillo, J. (2009). Origen, espacio y niveles de participación ciudadana. Daena: International Journal of Good Conscience, 179-193.

Hernández, R; Fernández, C; Baptista, P. (2004) Reseña de "Metodología de la investigación" Revista: Persona 2004. Tercera Edición.
Illera, M. d. (Julio de 2005). Convivencia y cultura ciudadana: Dos pilares fundamentales del derecho policivo. Revista de Derecho, 240-259.

Mockus, A. (2001). Armonizar ley, moral y cultura. Cultura ciudadana, prioridad de gobierno co resultados en prevención y control de violencia en Bogotá, 1995-1997.

Rico, J. L. (2011). "Estudio de Cultura Ciudadana Ciénaga, Magdalena 2011" (No publicado).

Rojo, M. A. (2009). Servicios públicos y cultura ciudadana.

Ruíz, J. I. (2007). Cultura ciudadana: Sus dimenciones psicosociales. Cuadernos Hispanoamericanos de Psicologia, 59-76.

Ruiz, J. I. (2010). Eficacia colectiva, cultura ciudadana y victimización: un análisis exploratorio sobre sus relaciones con diversas medidas del miedo al crimen. Acta Colombiana de Psicología, 103-114.

Ruiz, J. I., \& Trucios, L. A. (2009). Percepción de seguridad, victimización y cultura ciudadana: Pensamiento Psicológico, Vol 6. No 13. 193-202.

Zambrano, C. V. (2010). La diversidad cultural, los derechos culturales y la gestión ciudadana. Virajes, 183-201. 